\title{
Seronegative patients form a distinctive subgroup of immune-mediated necrotizing myopathy
}

Johan Lim, MD, Anke Rietveld, MD, Jan L. De Bleecker, PhD, Umesh A. Badrising, PhD, Christiaan G.J. Saris, PhD, Anneke J. van der Kooi, PhD,* and Marianne de Visser, PhD*

Neurol Neuroimmunol Neuroinflamm 2019;6:e513. doi:10.1212/NXI.0000000000000513

\section{Abstract}

\section{Objectives}

To investigate the characteristics of different clinico-serologic subgroups of immune-mediated necrotizing myopathy (IMNM).

\section{Methods}

We retrospectively reviewed data from medical charts of 64 patients diagnosed with IMNM between 2012 and 2017 in 3 neuromuscular referral centers in The Netherlands and 1 in Belgium.

\section{Results}

Seventeen patients had anti-3-hydroxy-3-methylglutaryl-coenzyme A reductase (HMGCR) autoantibodies (Abs), of whom 11 had a history of statin use, 15 had anti-signal recognition particle (SRP) Abs, 2 had anti-melanoma differentiation-associated protein 5 (MDA5) Abs, 22 patients were seronegative, and 9 patients did not have a complete $\mathrm{Ab}$ assessment. Moderate to severe disability in HMGCR Ab-positive and anti-SRP Ab-positive IMNM was common (71\% and $60 \%$, respectively) despite multimodality treatment. Compared with statin-associated antiHMGCR Ab-positive IMNM, statin-naive anti-HMGCR Ab-positive IMNM patients were more often men ( $67 \%$ vs $45 \%$ ), had lower rates of dysphagia ( $17 \%$ vs $45 \%$ ), and more frequently had third-line therapy ( $50 \%$ vs $9 \%$ ) and poor to fatal outcome ( $50 \%$ vs $0 \%$ ). Compared with seropositive IMNM, seronegative IMNM was characterized by female predominance (1:3), frequent occurrence of associated connective tissue disorders (22\% vs $9 \%$ ), and significantly higher rates of extramuscular disease activity (50\% vs $16 \%, p$ 0.014; 2 -sided Fisher exact), also after excluding patients with an associated connective tissue disease (35\% vs 7\%, $p$ 0.038; 2-sided Fisher exact).

\section{Conclusions}

Our findings indicate that seronegative IMNM forms a subgroup with distinctive features from seropositive IMNM.

\author{
Correspondence \\ Dr. Lim \\ j.lim@amc.nl
}




\section{Glossary}

$\mathbf{A b}=$ autoantibody; $\mathbf{C K}=$ creatine kinase; $\mathbf{C T D}=$ connective tissue disease; $\mathbf{D M}=$ dermatomyositis; EMA = extramuscular disease activity; HMGCR = hydroxy-3-methylglutaryl-coenzyme A reductase; IIM = idiopathic inflammatory myopathy; IMNM = immune-mediated necrotizing myopathy; ILD = interstitial lung disease; IVIg = intravenous immunoglobulin; MAA = myositis-associated Ab; MSA = myositis-specific autoantibody; SLE = systemic lupus erythematosus; $\mathbf{S R P}=$ signal recognition particle; SSc $=$ systemic sclerosis; ULN = upper limit of normal.

A majority of patients with immune-mediated necrotizing myopathy (IMNM) — a relatively new entity within the spectrum of idiopathic inflammatory myopathies (IIMs) suffer from disability despite treatment, indicating inadequate management. ${ }^{1-7}$ Indeed, disease management is complicated due to the heterogeneity of IMNM. On the one hand, key features of IMNM - i.e., progressive and often severe proximal muscle weakness-are generally shared among patients. ${ }^{5,8}$ On the other hand, disease manifestations such as dysphagia, axial and respiratory muscle weakness, and cancer vary significantly between patients. ${ }^{1-9}$ No clear data exist with regard to the variance in extramuscular disease activity (EMA) or associated connective tissue diseases (CTDs) between different patients with IMNM.

Progress in further characterizing IMNM has been made by the discovery of 2 myositis-specific autoantibodies (MSAs), i.e., anti-3-hydroxy-3-methylglutaryl-coenzyme A reductase (HMGCR) and anti-signal recognition particle (SRP) autoantibodies (Abs), which allowed for a serologic subclassification of IMNM. ${ }^{6,10}$ This has resulted in the identification of 2 subgroups of patients with IMNM with distinct clinico-serologic profiles. However, in a third of IMNM patients, these MSAs cannot be detected in sera and are therefore called "seronegative" IMNM. ${ }^{7}$ This group has not yet been characterized in detail, except for an association with cancer. ${ }^{9}$ We conducted a cross-sectional study to investigate clinical and serologic characteristics in a case series of IMNM, including seronegative IMNM, diagnosed in tertiary referral hospitals in The Netherlands and Belgium.

\section{Methods}

\section{Standard protocol approvals, registrations, and patient consents}

We retrospectively reviewed data from medical charts of patients diagnosed with IMNM between 2012 and 2017 in 3 neuromuscular referral centers in The Netherlands and 1 in Belgium. Diagnosis was based on the European Neuromuscular Centre criteria. ${ }^{7,11}$ Adult patients with subacute, symmetric, and predominantly proximal muscle weakness and a muscle biopsy characterized by muscle fiber necrosis and sparse to no inflammatory infiltrates were included. Toxic myopathy, active endocrinopathy, amyloidosis, family history of muscular dystrophy or proximal motor neuropathies, and any histopathologic features characteristic of other IIM subtypes were exclusion criteria. Furthermore, we excluded patients with anti-synthetase syndrome. ${ }^{12}$ Muscle biopsies (including immunostains) were re-evaluated in case of diagnostic uncertainty. ${ }^{7,11}$ The study was conducted in accordance with the Declaration of Helsinki and the research codes provided by the regional review board guidelines.

A predefined set of data was extracted from the medical charts: presence of MSAs and/or myositis-associated Abs (MAAs), age at onset, sex, duration of follow-up, history of statin use, the presence of proximal muscle weakness and dysphagia, EMA, the presence of CTDs or cancer, serum creatine kinase (CK) activity at diagnosis, treatment, and outcome. The following MSAs were assessed by a commercial semiquantitative line blot essay (Euroimmun, Lubeck, Germany): anti-Mi2 a and $\beta$, anti-transcriptional intermediary factor $1 \gamma$ (TIF $1 \gamma)$, anti-melanoma differentiation-associated protein 5 (MDA5), anti-nuclear matrix protein 2 (NXP2), anti-small ubiquitin-like modifier activating enzyme 1 (SAE1), anti-histidyl-tRNA synthetase (Jo1), anti-SRP, anti-threonyl-tRNA synthetase (PL7), anti-alanyl-tRNA synthetase (PL12), anti-glycyl-tRNA synthetase (EJ), and anti-isoleucyl-tRNA synthetase (OJ). On the same line blot assay, the following MAAs were assessed: anti-Ku, anti-polymyositis-scleroderma 100 protein (PMScl100), anti-polymyositis-scleroderma 75 protein (PMScl75), and anti-Ro52. ${ }^{13}$ A negative or a weak titer (1+) was considered negative, whereas moderately elevated $(2+)$ and high titers $(3+)$ were considered positive. In addition, antiHMGCR Abs were assessed by a commercial quantitative enzyme-linked immunosorbent assay (ELISA; INOVA, San Diego, CA). ${ }^{14}$ A level of anti-HMGCR Abs of 20 units/mL or higher was considered positive. The participating centers used the same line blot and anti-HMGCR assays. Patients were subsequently serologically grouped as follows: (1) antiHMGCR Ab-positive IMNM, further subclassified in statinassociated and statin-naive disease; (2) anti-SRP Ab-positive IMNM; (3) IMNM with MSAs other than anti-HMGCR or anti-SRP Abs; (4) seronegative IMNM in case no MSAs were found; and (5) IMNM with incomplete serologic status in case serum could not be analyzed by both the line blot essay and the quantitative anti-HMGCR ELISA. If a patient had a combination of MSAs and MAAs, the patient was classified into the respective MSA subgroup. EMA included interstitial lung disease (ILD), arthritis, Raynaud phenomenon, and skin or cardiac symptoms if considered IIM related. ${ }^{15}$ An associated CTD was considered present if a formal diagnosis of CTD was established at any time during the disease. Cancer was considered disease related if present from 3 years before to 3 years 
after a diagnosis of IMNM. ${ }^{9}$ Serum CK activity was expressed as the number of times the upper limit of normal (ULN) of the local laboratory. The ULN for serum CK activity of the Dutch laboratories was $145 \mathrm{IU} / \mathrm{L}$ for women and $171 \mathrm{IU} / \mathrm{L}$ for men, and in the Belgian laboratory, this was $170 \mathrm{IU} / \mathrm{L}$ for women and $195 \mathrm{IU} / \mathrm{L}$ for men. Treatment was scored as follows: (1) glucocorticoid monotherapy; (2) second-line therapy consisting of azathioprine and/or methotrexate and/or calcineurin inhibitors and/or mycophenolate mofetil; and (3) third-line therapy consisting of intravenous immunoglobulins (IVIgs), cyclophosphamide, rituximab, or other biologicals. Outcome was defined as good to excellent in case of normal or nearnormal functioning (with no apparent disability), moderate in case of slight to moderate disability (e.g., use of cane), poor in case of severe disability (e.g., wheelchair-bound), or dead.

\section{Statistical methods}

Results were primarily described using simple descriptive statistics. In addition, statistical tests for comparison of clinically relevant disease characteristics between seropositive and seronegative patients were performed, consisting of a MannWhitney $U$ test for age and a 2-sided Fisher exact test for sex, the presence of dysphagia, EMA, associated CTDs, the use of third-line treatment, and favorable outcome. The explorative nature of this study did not allow for multiple comparisons. ${ }^{16}$

\section{Data availability}

Any data not published within the article will (after anonymization) be shared on request from any qualified investigator.

\section{Results}

In total, 64 patients were included: 33 from Amsterdam, 16 from Ghent, 12 from Nijmegen, and 3 from Leiden. In 1 patient, the diagnosis was based on the presence of antiHMGCR Abs without pathologic confirmation. All other patients had undergone a muscle biopsy showing necrotizing myopathy with no or minimal lymphocytic infiltrates.

Seventeen patients (27\%) had anti-HMGCR Abs, of whom eleven $(65 \%)$ had a history of statin use. Fifteen patients $(23 \%)$ had anti-SRP Abs, of whom 1 patient (7\%) also had another MSA (anti-MDA5) and 3 patients (20\%) also had MAAs (antiRo52 Abs, anti-polymyositis-scleroderma-75 [PM-SCl75] Abs, and anti-Sjögren syndrome-A Abs, respectively). One patient (2\%) had anti-MDA5 Abs only. Twenty-two patients (34\%) had no MSAs, of whom 4 (18\%) had MAAs: 2 anti-Ku Abs, one anti-Ro52 Ab, and one anti-PM-Scl75 Ab. Nine patients (14\%) had incomplete Abs data: data on myositis line blot assay and anti-HMGCR Abs ELISA were not available in 7 and 2 patients, respectively. These 9 patients were excluded from further analysis. The demographics and disease characteristics of the remaining 55 patients are shown in table 1 .

Median age at onset was 54 years (range 16-82), and 33 patients $(61 \%)$ were women. The age at onset of patients with seronegative IMNM (median age 51 years, range 16-79 years) was comparable to that of patients with anti-SRP Ab-positive IMNM (median age 49 years, range 20-82 years), but lower than that of patients with HMGCR Ab-positive IMNM (median age 60 years, range 54-74 years). No statistical significant difference in age between seropositive and seronegative patients ( $p$ 0.189; Mann-Whitney $U$ test) was found.

Female preponderance was observed in seronegative IMNM (M:F ratio 1:3) compared with patients with seropositive IMNM (M:F ratio 1:1). This finding did not reach statistical significance ( $p 0.158 ; 2$-sided Fisher exact test). Median follow-up was 2 years (range 1 week to 24 years). The patient with a 1-week follow-up died of respiratory weakness and myocardial infarction. In total, 5 patients had a follow-up duration of less than 1 year.

All patients presented with proximal muscle weakness consisting of upper arm and upper leg weakness (legs more than arms), except 1 patient who had only proximal leg weakness. Dysphagia was present in 21 patients (38\%). Compared with statin-associated HMGCR Ab-positive (45\%) and anti-SRP $\mathrm{Ab}$-positive (53\%) IMNM, dysphagia was found less frequently in statin-naive anti-HMGCR Ab-positive (17\%) IMNM and seronegative (27\%) IMNM. No statistical significant difference in the prevalence of dysphagia between seropositive and seronegative patients $(p 0.262 ; 2$-sided Fisher exact test) was found.

EMA and associated CTDs were found predominantly in seronegative IMNM. Eight patients (15\%) of whom 5 (67\%) with seronegative IMNM had an associated CTD. Four patients had systemic sclerosis (SSc), 2 patients mixed CTD, 1 patient systemic lupus erythematosus (SLE), and 1 patient SLE and Sjögren's syndrome. EMA was found in 11 patients (50\%) with seronegative IMNM compared with 5 patients (16\%) with seropositive IMNM, respectively (table 1). A statistical significant difference in the occurrence of EMA ( $p$ 0.014; 2-sided Fisher exact test) but not in CTDs ( $p$ 0.248; 2 -sided Fisher's exact) between seropositive and seronegative patients was demonstrated, also after excluding 8 patients with an associated CTD ( $p 0.038$; 2 -sided Fisher exact test).

Among all included patients, the most frequent EMA features included arthritis in $6(11 \%)$, Raynaud phenomenon in 5 (9\%), and ILD in 4 (7\%). ILD was found in 3 patients (14\%) with seronegative IMNM and in 1 patient (6\%) with antiHMGCR Ab-positive IMNM. None of the patients had classical skin abnormalities consistent with dermatomyositis (DM), i.e., heliotropic erythema or Gottron papules. Related cancers were found in 5 cases (9\%), equally distributed among the subgroups. Related cancers included larynx carcinoma, ovary carcinoma, mediastinal tumor, bladder carcinoma, and breast carcinoma.

Median serum CK activity was 41 times the ULN, ranging from normal to 300 times the ULN. There was 1 patient with normal serum CK activity presenting with rapidly progressive 
Table 1 Demographics and disease characteristics of 55 patients with an IMNM

\begin{tabular}{|c|c|c|c|c|c|}
\hline & \multicolumn{2}{|c|}{ Anti-HMGCR $(n=17)$} & \multirow[b]{2}{*}{$\begin{array}{l}\text { Anti-SRP } \\
(n=15)\end{array}$} & \multirow[b]{2}{*}{$\begin{array}{l}\text { Anti-MDA5 } \\
(n=1)\end{array}$} & \multirow[b]{2}{*}{$\begin{array}{l}\text { Seronegative } \\
(n=22)\end{array}$} \\
\hline & $\begin{array}{l}\text { Statin +ve } \\
(n=11)\end{array}$ & $\begin{array}{l}\text { Statin -ve } \\
(n=6)\end{array}$ & & & \\
\hline \multicolumn{6}{|l|}{ Demographics } \\
\hline Age at onset ${ }^{a}$ (median years, range) & $63(54-74)$ & $57(52-65)$ & $49(20-82)$ & 47 & $51(16-79)$ \\
\hline Females $^{\mathrm{a}}(\mathrm{n}, \%)$ & $5(45)$ & $2(33)$ & $8(53)$ & $1(100)$ & $16(73)$ \\
\hline \multicolumn{6}{|l|}{ Disease features } \\
\hline Proximal muscle weakness ( $n, \%)$ & $11(100)$ & $6(100)$ & $15(100)$ & $1(100)$ & $21(95)$ \\
\hline Dysphagia $^{a}(n, \%)$ & $5(45)$ & $1(17)$ & $8(53)$ & $1(100)$ & $6(27)$ \\
\hline CK (median deviation from ULN, range) & $64(6-114)$ & $90(4-176)$ & $59(18-195)$ & 4 & $30(0-83)$ \\
\hline Extramuscular disease $^{\mathrm{b}}$ activity $(\mathrm{n}, \%)$ & $1(9)$ & $1(17)$ & $3(20)$ & $0(0)$ & $11(50)$ \\
\hline $\mathrm{CTD}^{\mathrm{a}, \mathrm{c}}(\mathrm{n}, \%)$ & $0(0)$ & $1(17)$ & $2(13)$ & $0(0)$ & $5(22)$ \\
\hline MAAs (n, \%) & $0(0)$ & $0(0)$ & $3(20)$ & $0(0)$ & $4(18)$ \\
\hline Cancer (n, \%) & $1(9)$ & $1(17)$ & $1(7)$ & $0(0)$ & $2(9)$ \\
\hline
\end{tabular}

Abbreviations: anti-MDA5 = anti-melanoma differentiation-associated protein 5 Ab-positive IMNM; anti-SRP = anti-signal recognition particle Ab-positive IMNM; CK = serum creatine kinase activity; $C T D$ = connective tissue disease; IMNM = immune-mediated necrotizing myopathy; MAA = myositis-associated Ab; seronegative = IMNM without any myositis-specific Ab; statin +ve anti-HMGCR = statin-associated anti-3-hydroxy-3-methylglutaryl-CoA reductase (HMGCR) autoantibody (Ab)-positive IMNM; statin -ve anti-HMGCR = statin-naive anti-HMGCR Ab-positive IMNM; ULN = upper limit of normal.

${ }^{a}$ The differences in age, sex, and the presence of dysphagia or associated CTDs between seropositive and seronegative patients were not statistically significant.

b Interstitial lung disease, arthritis, Raynaud phenomenon, and skin or cardiac symptoms if considered myositis related. The difference in the prevalence of EMA between seropositive and seronegative patients was statistically significant (16\% vs 50\%, $p 0.014 ; 2$-sided Fisher exact test), also after excluding patients with an associated connective tissue disease (7\% vs 35\%, $p$ 0.038; 2 -sided Fisher exact test).

' Five patients with systemic sclerosis, 3 patients with mixed connective tissue disease, 1 case with systemic lupus erythematosus (SLE), and 1 case with both SLE and Sjögren syndrome.

muscle weakness and polyarthritis, who had a muscle biopsy consistent with IMNM, no MSAs/MAAs, and responded favorably to glucocorticoid therapy. Differences in median serum CK activity did not differ between the 3 IMNM subgroups.

Eleven patients $(20 \%)$ received glucocorticoid monotherapy, whereas 28 (51\%) and 14 (25\%), respectively, had secondand third-line treatment (table 2). Treatment data were not available for 2 patients (4\%). Third-line therapy, mostly IVIg, was administered to $50 \%$ of the statin-naive anti-HMGCR $\mathrm{Ab}$-positive IMNM patients and 55\% of the anti-SRP $\mathrm{Ab}-$ positive IMNM patients, as compared to $10 \%$ of the statinassociated anti-HMGCR Ab-positive IMNM patients and $15 \%$ of seronegative IMNM patients. We found no statistical significant differences in the rates of third-line treatment between seropositive and seronegative patients ( $p 0.200 ; 2$-sided Fisher exact test).

Twenty-one patients (38\%) had good to excellent outcome, 26 patients (47\%) had moderate outcome, 3 patients (5\%) had poor outcome, and 4 patients (7\%) died (table 2). Outcome data were not available in 1 patient $(2 \%)$. Causes of deaths included laryngeal carcinoma, metastasized bladder carcinoma, renal failure, and a mediastinal tumor. Except for the small group of statin-naive anti-HMGCR Ab-positive
IMNM patients, of whom 3 (50\%) had a poor outcome or died, outcome was essentially the same across the other subgroups. We found no statistical significant differences in the rates of good-excellent outcomes between seropositive and seronegative patients ( $p 0.397 ; 2$-sided Fisher exact test).

\section{Discussion}

We found that seronegative IMNM had distinctive features including a significantly higher frequency of EMA. The higher rates of EMA in seronegative IMNM were not fully explained by the concomitant presence of an associated CTD, thus indicating a truly distinctive feature. A female predominance and higher rates of associated connective tissue disorders were seen in seronegative patients, but these findings did not reach statistical significance, probably due to the relatively small sample size. One study found higher rates of cancer in seronegative IMNM compared with the HMGCR Ab-positive and anti-SRP Ab-positive IMNM. ${ }^{9}$ We could not confirm this in our study, again probably because of the relatively small sample size of our study. It may well be that the female preponderance has to be ascribed to the association with CTDs as was the case in two-thirds of the seronegative patients. SSc was the most frequent CTD in these patients. A recent study found that necrotizing myopathy was the second most 
Table 2 Treatment and outcome in 55 patients with an immune-mediated necrotizing myopathy (IMNM)

\begin{tabular}{|c|c|c|c|c|c|}
\hline & \multicolumn{2}{|l|}{ Anti-HMGCR $(n=17)$} & \multirow{2}{*}{$\begin{array}{l}\text { Anti-SRP } \\
(n=15)\end{array}$} & \multirow{2}{*}{$\begin{array}{l}\text { Anti-MDA5 } \\
(n=1)\end{array}$} & \multirow{2}{*}{$\begin{array}{l}\text { Seronegative } \\
(n=22)\end{array}$} \\
\hline & Statin +ve $(n=11)$ & Statin -ve $(n=6)$ & & & \\
\hline \multicolumn{6}{|l|}{ Treatment $^{a}$} \\
\hline Glucocorticoid monotherapy (n, \%) & $2(18)$ & $1(17)$ & $3(20)$ & $0(0)$ & $5(23)$ \\
\hline Second-line therapy $(n, \%)$ & $8(73)$ & $2(33)$ & $5(33)$ & $1(100)$ & $12(54)$ \\
\hline Third-line therapy $(n, \%)$ & 1 (9) & $3(50)$ & $7(47)$ & $0(0)$ & $3(14)$ \\
\hline \multicolumn{6}{|l|}{ Outcome $^{\text {b }}$} \\
\hline Good-excellent outcome (n, \%) & $4(36)$ & $1(17)$ & $6(35)$ & $0(0)$ & $10(45)$ \\
\hline Moderate outcome $(n, \%)$ & $7(63)$ & $2(33)$ & $8(57)$ & $1(100)$ & $8(36)$ \\
\hline Poor outcome $(n, \%)$ & $0(0)$ & $2(33)$ & $0(0)$ & $0(0)$ & $1(5)$ \\
\hline Dead (n, \%) & $0(0)$ & $1(17)$ & $1(7)$ & $0(0)$ & $2(9)$ \\
\hline \multicolumn{6}{|c|}{$\begin{array}{l}\text { Abbreviations: anti-MDA5 = anti-melanoma differentiation-associated protein } 5 \text { Ab-positive IMNM; anti-SRP = anti-signal recognition particle Ab-positive } \\
\text { IMNM; seronegative = IMNM without any myositis-specific Ab; statin +ve anti-HMGCR = statin-associated anti-3-hydroxy-3-methylglutaryl-CoA reductase } \\
\text { (HMGCR) autoantibody (Ab)-positive IMNM; statin -ve anti-HMGCR = statin-naive anti-HMGCR Ab-positive IMNM. } \\
\text { a Treatment schemes of patients with IMNM shown in percentages. Treatment data from } 2(9 \%) \text { patients with seronegative IMNM are missing; azathioprine } \\
\text { and/or methotrexate and/or mycophenolate mofetil were considered second-line therapy; intravenous immunoglobulins ( } \mathrm{n}=13 \text { ) and/or rituximab }(\mathrm{n}=3 \text {; all } \\
\text { in combination with intravenous immunoglobulins) and/or cyclofosfamide }(\mathrm{n}=1 \text { ) were considered third-line therapy. Differences in the rates of third-line } \\
\text { treatment between seropositive and seronegative patients were not statistically significant. } \\
\text { b Outcomes in IMNM shown in percentages. Outcome data from } 1(5 \%) \text { patient with seronegative IMNM is missing; good-excellent outcome was defined as } \\
\text { normal or near-normal functioning with no apparent disability; moderate outcome was defined as slight to moderate disability (e.g., use of cane); poor } \\
\text { outcome was defined as severe disability (e.g., wheelchair-bound); causes of deaths included laryngeal carcinoma, metastasized bladder carcinoma, } \\
\text { pneumonia, respiratory weakness and myocardial infarction, peritonitis, and a mediastinal tumor. Differences in the rates of good-excellent outcomes } \\
\text { between seropositive and seronegative patients were not statistically significant. }\end{array}$} \\
\hline
\end{tabular}

common histologic subtype (according to the 2004 ENMC criteria) in patients with SSc. ${ }^{17}$

Of note, 2 patients had anti-MDA5 Ab-positive IMNM. These antibodies are associated with classic DM or overlap myositis with other cutaneous manifestations, frequent occurrence of prominent ILD, and relatively little myositis. ${ }^{5,18}$ The patient with solely anti-MDA5 Abs has been previously described and had no skin rash or ILD. ${ }^{19}$ The patient with both anti-SRP and anti-MDA5 Abs presented with proximal muscle weakness, nonspecific skin erythema on her chest and upper arms, digital cutaneous ulceration, arthralgia, and a serum CK activity of 90 times the ULN. This concomitant presence of 2 MSAs is remarkable because usually, MSAs are mutually exclusive. ${ }^{4,6,20}$

We found some apparent differences when we compared our seropositive patients with those reported in previous reports. Although earlier reports did not find any differences between statin-associated and statin-naive anti-HMGCR Ab-positive IMNM except for younger age at onset, higher serum CK activity, and more frequent non-Caucasian ethnicity in the latter, we observed that statin-naive anti-HMGCR $\mathrm{Ab}-$ positive IMNM patients were more often men ( $66 \%$ vs $45 \%)$, had lower rates of dysphagia ( $17 \%$ vs $46 \%$ ), received more often third-line therapy ( $50 \%$ vs $9 \%$ ), and had more frequently poor to fatal outcome ( $50 \%$ vs $0 \%) .^{6}$ The number of cases with a history of statin use in anti-HMGCR-positive IMNM (65\%) was comparable to that found in North
American and European case series with HMGCR Abpositive IMNM (44\%-67\%) and much higher as compared to a Japanese case series with HMGCR Ab-positive IMNM $(18 \%) .{ }^{1,2,6}$ None of our anti-SRP Ab-positive IMNM patients had ILD, although an association in $13 \%-22 \%$ has been reported. ${ }^{1,3,4}$ The frequency of cancer in our patients was too low to compare it with that of other case series. Our data confirm that in general, HMGCR Ab-positive IMNM and anti-SRP IMNM are severe conditions, as illustrated by the proportion of patients $(65 \%)$ with moderate, severe, or fatal outcome despite multimodality treatment. ${ }^{1-4,6}$

The strength of our study is the detailed description of the IMNM group as a whole, and in particular with respect to the description of the as yet relatively underexposed seronegative patients. The main limitation of our study is the retrospective nature and the relatively small sample size of the study.

Our findings indicate that seronegative IMNM is distinct from seropositive IMNM, given the frequent occurrence of EMA and associated CTDs and the female preponderance in the former. The results may not necessarily be extrapolated to other ethnic populations, considering the predominantly European ancestry of our study population. Larger prospective studies are warranted to confirm our findings.

\section{Author contributions}

J. Lim: drafting/revising the manuscript, study concept or design, analysis or interpretation of data, and accepts 
responsibility for conduct of research and final approval. A. Rietveld, J.L. De Bleecker, U.A. Badrising, and C.G.J. Saris: drafting/revising the manuscript, analysis or interpretation of data, and accepts responsibility for conduct of research and final approval. A.J. van der Kooi and M. de Visser: drafting/ revising the manuscript, study concept or design, analysis or interpretation of data, and accepts responsibility for conduct of research and final approval.

\section{Acknowledgment}

The authors thank Dr. Eleonora Aronica for her help with the revision of muscle biopsies, Dr. Ester van Leeuwen for performing the serological assessment, and Dr. Rob de Haan for statistical consultation.

\section{Study funding}

No targeted funding reported.

\section{Disclosure}

J. Lim reports no disclosures. A. Rietveld received research support from Prinses Beatrix Spierfonds. J.L. De Bleecker served on the scientific advisory board of Sanofi/Genzyme, Pfizer, and CSL Behring and received travel funding and/or speaker honoraria from Sanofi/Genzyme and CSL Behring. U.A. Badrising consulted for Novartis and Argenx (compensation paid to Leiden University Medical Center). C.G.J. Saris reports no disclosures. A.J. van der Koii received research support from CSL Behring. M. de Visser served on the scientific advisory board of AveXis; served on the editorial board of Neuromuscular Disorders and Clinical Neurology and Neurosurgery; and is a member of the adjudication committee for Bristol Myers. Full disclosure form information provided by the authors is available with the full text of this article at Neurology.org/NN.

Received May 7, 2018. Accepted in final form August 15, 2018.

\section{References}

1. Watanabe Y, Uruha A, Suzuki S, et al. Clinical features and prognosis in anti-SRP and anti-HMGCR necrotising myopathy. J Neurol Neurosurg Psychiatry 2016;87: 1038-1044.

2. Allenbach Y, Drouot L, Rigolet A, et al. Anti-HMGCR autoantibodies in European patients with autoimmune necrotizing myopathies: inconstant exposure to statin. Medicine 2014;93:150-157.

3. Pinal-Fernandez I, Parks C, Werner JL, et al. Longitudinal course of disease in a large cohort of myositis patients with autoantibodies recognizing the signal recognition particle. Arthritis Care Res 2017;69:263-270.

4. Suzuki S, Nishikawa A, Kuwana M, et al. Inflammatory myopathy with anti-signal recognition particle antibodies: case series of 100 patients. Orphanet J Rare Dis 2015;10:61.

5. Allenbach Y, Benveniste O, Goebel HH, Stenzel W. Integrated classification of inflammatory myopathies. Neuropathol Appl Neurobiol 2017;43:62-81.

6. Mammen AL, Chung T, Christopher-Stine L, et al. Autoantibodies against 3-hydroxy3-methylglutaryl-coenzyme A reductase in patients with statin-associated autoimmune myopathy. Arthritis Rheum 2011;63:713-721.

7. Allenbach $\mathrm{Y}, \mathrm{Mammen} \mathrm{AL}$, Benveniste O, Stenzel W; Immune-mediated Necrotizing Myopathies Working Group. 224th ENMC International Workshop: clinico-seropathological classification of immune-mediated necrotizing myopathies Zandvoort, The Netherlands, 14-16 October 2016. Neuromuscul Disord 2018;28:87-99.

8. Basharat $\mathrm{P}$, Christopher-Stine L. Immune-mediated necrotizing myopathy: update on diagnosis and management. Curr Rheumatol Rep 2015;17:72.

9. Allenbach Y, Keraen J, Bouvier AM, et al. High risk of cancer in autoimmune necrotizing myopathies: usefulness of myositis specific antibody. Brain 2016;139:2131-2135.

10. Targoff IN, Johnson AE, Miller FW. Antibody to signal recognition particle in polymyositis. Arthritis Rheum 1990;33:1361-1370.

11. Hoogendijk JE, Amato AA, Lecky BR, et al. 119th ENMC International Workshop: trial design in adult idiopathic inflammatory myopathies, with the exception of in clusion body myositis, 10-12 October 2003, Naarden, The Netherlands. Neuromuscul Disord 2004; 14:337-345

12. Uruha A, Suzuki S, Suzuki N, et al. Perifascicular necrosis in anti-synthetase syndrome beyond anti-Jo-1. Brain 2016;139:e50.

13. Cavazzana I, Fredi M, Ceribelli A, et al. Immunoprecipitation assays in 57 myositis sera. J Immunol Methods 2016;433:1-5.

14. Shovman O, Gilburd B, Chayat C, et al. Anti-HMGCR antibodies demonstrate high diagnostic value in the diagnosis of immune-mediated necrotizing myopathy following statin exposure. Immunol Res 2017;65:276-281.

15. Miller FW, Rider LG, Chung YL, et al. Proposed preliminary core set measures for disease outcome assessment in adult and juvenile idiopathic inflammatory myopathies. Rheumatology 2001;40:1262-1273.

16. Rothman KJ. No adjustments are needed for multiple comparisons. Epidemiology 1990; $1: 43-46$.

17. Paik JJ, Wigley FM, Lloyd TE, et al. Spectrum of muscle histopathologic findings in forty-two scleroderma patients with weakness. Arthritis Care Res 2015;67:1416-1425.

18. Senecal JL, Raynauld JP, Troyanov Y. Editorial: a new classification of adult autoimmune myositis. Arthritis Rheumatol 2017;69:878-884.

19. Jaeger B, de Visser M, Aronica E, van der Kooi AJ. Respiratory failure as presenting symptom of necrotizing autoimmune myopathy with anti-melanoma differentiationassociated gene 5 antibodies. Neuromuscul Disord 2015;25:457-460.

20. Betteridge Z, McHugh N. Myositis-specific autoantibodies: an important tool to support diagnosis of myositis. J Intern Med 2016;280:8-23. 


\title{
Neurology \\ Neuroimmunology \& Neuroinflammation
}

\author{
Seronegative patients form a distinctive subgroup of immune-mediated necrotizing \\ myopathy \\ Johan Lim, Anke Rietveld, Jan L. De Bleecker, et al. \\ Neurol Neuroimmunol Neuroinflamm 2019;6; \\ DOI 10.1212/NXI.0000000000000513
}

This information is current as of October 16, 2018

\begin{abstract}
Updated Information \& Services

References

Citations

Subspecialty Collections

Permissions \& Licensing

Reprints

including high resolution figures, can be found at: http://nn.neurology.org/content/6/1/e513.full.html

This article cites 20 articles, 1 of which you can access for free at: http://nn.neurology.org/content/6/1/e513.full.html\#\#ref-list-1

This article has been cited by 1 HighWire-hosted articles: http://nn.neurology.org/content/6/1/e513.full.html\#\#otherarticles

This article, along with others on similar topics, appears in the following collection(s):

Autoimmune diseases

http://nn.neurology.org//cgi/collection/autoimmune_diseases Muscle disease

http://nn.neurology.org//cgi/collection/muscle_disease

Information about reproducing this article in parts (figures,tables) or in its entirety can be found online at:

http://nn.neurology.org/misc/about.xhtml\#permissions

Information about ordering reprints can be found online:

http://nn.neurology.org/misc/addir.xhtml\#reprintsus
\end{abstract}

Neurol Neuroimmunol Neuroinflamm is an official journal of the American Academy of Neurology.

Published since April 2014, it is an open-access, online-only, continuous publication journal. Copyright

Copyright $\odot 2018$ The Author(s). Published by Wolters Kluwer Health, Inc. on behalf of the American

Academy of Neurology.. All rights reserved. Online ISSN: 2332-7812.



